\title{
Minicurso com roda de conversa como estratégia didática sobre opioides: relato de experiência
}

\author{
Adolfo Paulo de Mattos Júnior \\ Ana Sofia Martelli Chaib Saliba \\ Luiz Otávio Lourenço \\ Cibele Marli Cação Paiva Gouvêa ${ }^{1}$
}

Resumo: O uso e a dependência de drogas ilícitas constituem importante problema social e de saúde pública em todo o mundo, inclusive no Brasil. Dentre as substâncias utilizadas como medicamentos e de abuso destacam-se os opioides. Apesar de estudado pelos cursos da área de saúde, este assunto é pouco abordado nos cursos de graduação da área biológica. O grupo PET-X da Universidade $X$ ministrou minicurso, com roda de conversa sobre a atuação e consequências do uso de opioides nas diferentes fases da vida, como forma de contribuir para o conhecimento do assunto fora da sala de aula. Os resultados demonstraram que a maioria dos participantes não leu o material de apoio didático antes da realização do minicurso, tendo em vista que houve aumento significativo $(\mathrm{p}<0,05)$ de respostas corretas para o questionário aplicado após o minicurso, quando comparado ao aplicado antes da realização deste. A roda de conversa se mostrou muito eficiente para discussão do tema. Todos participaram do diálogo disparado pelas questões-tema e foi um momento único para sanar dúvidas, conhecer mais sobre o assunto e inclusive partilhar experiências próprias com o uso de opioides. No ensino superior as rodas de conversa encontram pouco espaço, contudo os resultados deste trabalho sugerem que seja uma estratégia didática concorre para a aquisição de conhecimento, valorizando aspectos importantes como a compreensão do tema, a escuta, o respeito, a construção e a reconstrução do conhecimento, o que contribui para a autonomia estudantil na construção do conhecimento.

Palavras chave: Derivados do ópio; Diálogo; Educação; Ensino-aprendizagem; PETBiologia.

Abstract: The use and dependence of illicit drugs are important social and public health problems around the world, including Brazil. One of the drug class used as medicines and of abuse are the opioids. Although studied for Healthcare courses, this subject is little discussed in the Biological courses. The PET-X group of the Universidade taught a short course, with conversation circle, about the opioids action mode and consequences of its use in different stages of life, as a way of contribute to the knowledge of the subject outside of the classroom. The results showed that most participants do not read the didactic support material before the completion of the course, considering that there was a significant increase $(\mathrm{p}<0.05)$ of correct answers to the questionnaire applied after the lecture, when compared to the applied

${ }^{1}$ Universidade Federal de Alfenas 
prior to it. The conversation circle turned out to be very efficient for the topic discussion. All of the course attendants participated in the dialogue raised by the theme issues and it proved to be a unique moment to address questions, learn more about it and even share own experiences with the use of opioids. In higher education the use of conversation circles is limited, however the results of this study suggest that it is a didactic strategy that contributes for the acquisition of knowledge and highlighted important aspects in the learning process, such as subject comprehension, attention and respect to others, construction and reconstruction of knowledge, important facts to student autonomy in the construction of knowledge.

Keywords: Opium derivatives; Dialogue; Education; Teaching-learning; PET-Biology.

\section{INTRODUÇÃO}

O uso e a dependência de drogas ilícitas constituem importante problema social e de saúde pública em todo o mundo e também no Brasil, pois apresenta alta prevalência e início cada vez mais precoce (JORGE et al., 2018; NAHAS et al., 2017; UNODC, 2017). Dentre as substâncias utilizadas como medicamentos e de abuso destacam-se os opioides, podendo ser naturais, que são os derivados do ópio como a morfina, a codeína e a tebaína; os semissintéticos, que resultam de modificação da substância original, sendo a heroína (diacetilmorfina) a primeira descrita na literatura médica e os sintéticos, que são fabricados em laboratório a partir da replicação da estrutura química dos opioides naturais, sendo a metadona a mais amplamente conhecida (PEREIRA; ANDRADE; TAKITANE, 2016).

Os opioides são os fármacos de escolha para o alívio da dor aguda e da dor oncológica intensa. Também têm sido utilizados no tratamento de diversas síndromes dolorosas crônicas não oncológicas. Embora o uso para a dor crônica não oncológica seja controverso, há evidências de benefícios para alguns grupos de pessoas. Entretanto, o grande temor do uso de opioides, apesar do excelente efeito analgésico, é a dependência (KRAYCHETE et al., 2014; NASCIMENTO; SAKATA, 2011; PEREIRA; ANDRADE; TAKITANE, 2016). Somam-se a isso outros fatores de risco para co-morbidades associadas à dependência de opioides, os efeitos adversos que podem afetar o desenvolvimento embrionário e fetal e a morte pelo excesso de substância, como a ocorrida com diversos artistas famosos (PEREIRA; ANDRADE; TAKITANE, 2016; WEBSTER, 2017). Em torno de $1,3 \%$ da população brasileira faz uso contumaz de derivados do ópio, principalmente, a

Revista Extensão em Foco, nº 19, Jul./ Dez. (2019), p. 104 - 114. 
heroína. Na América do Sul, entre 0,3 a 0,4 \% da população é usuário de opioides, sendo a maioria destes usuários as mulheres (UNODC, 2017).

Trata-se de tema relevante do ponto de vista biológico e social e ainda pouco estudado no ensino superior por cursos da área biológica. Desta forma, o presente trabalho, relata a experiência obtida pelo grupo do Programa de Educação Tutorial, PET-Biologia, da Universidade Federal de Alfenas (UNIFAL-MG, Alfenas, MG), decorrente da aplicação de um minicurso sobre opioides, para alunos de graduação e pós-graduação em cursos da área biológica.

O minicurso é uma estratégia de ensino, cujo objetivo é expor estruturas básicas e técnicas que atendam a diferentes temáticas, a fim de contribuir na formação acadêmica e propagar o conhecimento fora da sala de aula. Compreende-se que o uso de estratégias de ensino diferenciadas influencia de forma direta a obtenção de melhores resultados de aprendizagem. Além disso, apresenta potencial para fazer face à fragmentação, à linearidade e ao excessivo individualismo que, constantemente, permeiam os currículos dos cursos de graduação (CARVALHO; GIL-PÉREZ, 2011).

As rodas de conversa consistem em um método de participação coletiva de debate acerca de determinada temática em que é possível dialogar com os sujeitos, que se expressam e escutam seus pares e a si mesmos, por meio do exercício reflexivo. Um dos seus objetivos é de socializar saberes e implementar a partilha de experiências, de conversas, de divulgação e de conhecimentos entre os envolvidos, na perspectiva de construir e reconstruir novos conhecimentos sobre a temática proposta (WARSCHAUER, 2001; 2002). Para Freire (2011) uma das tarefas da escola, como centro de produção sistemática do conhecimento, é trabalhar, criticamente, a compreensão das coisas e dos fatos, assim como a sua comunicação.

$\mathrm{Na}$ educação escolar formal, sobretudo no ensino superior, a roda de conversa é pouco presente, devido a muitos fatores. Entre eles, a perspectiva de homogeneização, padronização e organização de espaços, tempos e currículos, estruturados de tal maneira a deixar poucas oportunidades para a manifestação das diferenças e singularidades, resultando em poucas possibilidades no cotidiano escolar para a inclusão do diverso (WARSCHAUER, 2004).

Revista Extensão em Foco, nº 19, Jul./ Dez. (2019), p. 104 - 114. 
A educação, assim como a qualidade do ensino são temas que levantam inúmeras discussões, visto que existem inúmeras estratégias educacionais, as quais produzem diferentes resultados de ensino e aprendizagem, de acordo com o local e grupo com o qual se trabalha, devido a diferença de cultura, idade, ambiente de estudo, entre outros (BORGES, 2002).

$\mathrm{O}$ ensino de processos biológicos, assim como de qualquer assunto relacionado à disciplina de biologia, quando realizado com a participação o aluno no processo de ensino e aprendizagem, como agente do seu aprendizado, permite que os indivíduos ampliem seus conhecimentos a respeito dos fenômenos que ocorrem no mundo e com os seres vivos e desenvolvam o pensamento crítico a respeito da importância da ciência e tecnologia na vida cotidiana (KRASILCHIK, 2008).

\section{MATERIAIS E MÉTODOS}

O grupo PET-X (Programa de Educação Tutorial) desenvolve atividades de ensino, pesquisa e extensão na Universidade $X$ desde 2009. Dentre estas atividades, destacam-se os minicursos, que abordam assuntos atuais e de interesse da comunidade acadêmica.

No segundo semestre de 2017 foi ministrado o minicurso intitulado "Consequências do uso de opioides durante a vida", durante a "V jornada da Biologia" realizada no Câmpus sede da Universidade X, em cidade (estado).

O minicurso contou com exposição oral do tema e roda de conversa. Foi também disponibilizado para download, material de apoio didático no sítio do grupo, na página da Universidade $\mathrm{X}$, oportunizando aos participantes o contato com o tema prévio ao minicurso. Antes do início e ao final do minicurso foram aplicados questionários aos participantes para avaliação do conhecimento sobre o tema abordado.

O minicurso teve duração de $4 \mathrm{~h}$, sendo dividido em parte teórica e prática. A parte teórica abordou aspectos introdutórios, histórico do uso de ópio e derivados e alguns efeitos causados por seu uso prolongado no desenvolvimento embrionário e fetal (no caso de uso pela gestante), na adolescência e na vida adulta. A parte teórica foi desenvolvida com a utilização de multimeios, tendo como base o material didático disponibilizado.

A roda de conversa teve início após a explanação teórica. Os participantes sentados em cadeiras ou onde se sentissem mais confortáveis foram convidados a discutir o tema em ambiente acolhedor e descontraído. Foi realizada dinâmica de apresentação dos participantes, 
seguida de explicação sobre a roda de conversa. Os participantes falaram, um por vez, o nome, o curso, quando ingressaram na Universidade e o que mais achassem propício para se apresentar, pois é importante que os participantes se conheçam e percebam pontos em comum, o que gera empatia e ajuda a criar vínculos de confiança, necessários para o diálogo. Algumas questões-tema foram projetadas, com o propósito de suscitar o início do diálogo e a partilha de idéias e experiências entre os participantes. Foram elas: Os opioides, mesmo quando utilizados para alívio da dor, podem causar dependência? De acordo com as indicações da escada da OMS para dores oncológicas, existem casos que exigem a aplicação de uma alta dose de opioides? O uso abrupto de antagonistas opioides pode causar dependência física? Qual(is) o(s) principal(is) ponto(s) a serem considerados para garantir melhores cuidados na gestação? Não há alterações no sistema reprodutor com o uso prolongado de opioides. Pessoas dependentes de opióides tornam-se mais vulneráveis a adquirir doenças transmitidas pelo sangue? Qual(is) seria(m) o(s) melhor(es) medicamento(s) para iniciar o tratamento de uma gestante? A probabilidade de se tornar dependente químico de opioides possui relação com a idade do usuário? Os efeitos dos opioides nos bebês permanecem apenas durante a gestação ou se prolongam pela vida? O uso de opioides antagonistas aumenta a concentração de LH? A fisiologia masculina é mais sensível aos opioides do que a feminina? Qual o mais notável efeito do abuso de ópio durante a gestação? A maioria das mortes causadas por opioides prescritos ocorre na faixa dos 60-70 anos? Usuários de cocaína possuem a maior probabilidade de se tornaram também dependentes de heroína? Você tem algum amigo usuário de ópio, o que você seria capaz de fazer para auxiliálo a passar por isso?

A partir desse questionamento inicial, todos foram convidados a conversar, a relatar e a trocar experiências. Os participantes iniciaram a conversa de forma tímida, mas, rapidamente, a conversa fluiu com naturalidade e as falas se intercruzavam na dinâmica própria da conversa. Foram 13 interlocutores, dos cursos de biologia, biomedicina e biotecnologia, dos níveis de graduação e de pós-graduação. Tendo em vista que a proposta foi de conversa, os ministrantes do minicurso se inseriram no contexto, para impulsionar o diálogo e também participar ativamente dele. A conversa desenvolveu-se com tanta espontaneidade que, no final, quando foi necessário encerrar a roda, devido ao término do tempo disponível para a atividade, todos lamentaram e alguns continuaram a conversa no hall externo à sala onde ocorreu o minicurso.

Revista Extensão em Foco, nº 19, Jul./ Dez. (2019), p. 104 - 114. 
Antes do início e ao final do minicurso os participantes responderam o questionário apresentado na Tabela 1, para avaliação dos conhecimentos prévios e após a realização do minicurso. Os questionários foram compostos por questões estruturadas, o que permitiu a avaliação quantitativa das respostas dadas. Para a análise dos dados foram utilizados os programas Microsoft Excel $^{\circledR}$ e GraphPadPrisma 7.0 ${ }^{\circledR}$. Os dados foram expressos em porcentagem de respostas corretas para cada questão e porcentagem total de respostas corretas antes e após a realização do minicurso. Os dados foram submetidos ao teste $t$ Student e considerados significativamente diferentes quando $\mathrm{p}<0,05$.

Tabela 1 - Questionário aplicado aos participantes, antes do início e após a realização do minicurso

\begin{tabular}{|l|l|l|l|}
\hline \multicolumn{2}{|c|}{ Questão } & \multicolumn{1}{|c|}{ Alternativas } & \multicolumn{1}{|c|}{$\begin{array}{c}\text { Resposta } \\
\text { correta }\end{array}$} \\
\hline $\begin{array}{l}\text { 1. Qual destes compostos não é um } \\
\text { opioide? }\end{array}$ & $\begin{array}{l}\text { a) Heroína } \\
\text { b) Morfina } \\
\text { c) Buprenorfina } \\
\text { d) Cocaína }\end{array}$ & D \\
\hline $\begin{array}{l}\text { 2. Em qual continente o ópio foi } \\
\text { primeiramente cultivado? }\end{array}$ & $\begin{array}{l}\text { a) América } \\
\text { b) Europa } \\
\text { c) África } \\
\text { d) Ásia }\end{array}$ & A \\
\hline $\begin{array}{l}\text { 3. Qual o nome dos três principais } \\
\text { receptores opioides? }\end{array}$ & $\begin{array}{l}\text { a) Mu, Kappa e Delta } \\
\text { b) Zeta, Kappa e Phi } \\
\text { c) Mu, Tetha e Kappa }\end{array}$ \\
\hline $\begin{array}{l}\text { Com relação aos neurônios } \\
\text { dopaminérgicos, os opioides: }\end{array}$ & $\begin{array}{l}\text { a) Promovem a liberação de } \\
\text { dopamina } \\
\text { b) Inibem a liberação de } \\
\text { dopamina }\end{array}$ & A \\
\hline
\end{tabular}

Fonte: Dados dos autores.

\section{RESULTADOS E DISCUSSÃO}

O minicurso é muito útil como estratégia de ensino, especialmente, de temas não contemplados no currículo e ainda ajuda a preencher as lacunas deixadas no ensino, como as causadas pela fragmentação de conteúdos, além de outros. A estratégia de roda de conversa pode ser usada para quase todo conteúdo, pois é fácil de ser ministrada e bem proveitosa, sendo o tempo disponível para a abordagem do tema, uma limitação ao seu uso. Nela o professor deve selecionar algumas questões-tema (relacionadas ao tema da aula), destacando Revista Extensão em Foco, nº 19, Jul./ Dez. (2019), p. 104 - 114. 
os pontos relevantes do conteúdo, a fim de propiciar um meio para o início do diálogo. É importante que haja um ambiente propício para o diálogo, em que todos possam se sentir à vontade para partilhar e escutar, de modo que o ato de falar seja relevante para o grupo e suscite a atenção na escuta. Nas rodas de conversa, o diálogo é um momento singular de partilha, porque pressupõe um exercício de escuta, momentos mais numerosos que os de fala, em que participam vários interlocutores. As colocações de cada participante são construídas por meio da interação com o outro, para complementar, discordar ou concordar com a(s) fala(s) anterior(es).

No presente trabalho os participantes interagiram com naturalidade, todos participaram e se interessaram por buscar respostas aos questionamentos, com base no conhecimento adquirido no minicurso. No desenvolvimento dos diálogos, confirmou-se o proposto por Warschauer (2002), que a roda de conversa é uma construção própria de cada grupo e constitui-se em um momento de diálogo, por excelência, em que ocorre a interação entre os participantes do grupo, sob a organização do coordenador. Para Shor e Freire (1987) o diálogo tem limites e contradições que condicionam o que se pode fazer, é um caminho para recriar o conhecimento, bem como para aprender. Para alcançar os objetivos de transformação, o diálogo implica em responsabilidade, direcionamento, determinação e disciplina.

Alguns participantes se sentiram confortáveis e socializaram a experiência própria com o uso de opioides, prescritos por médico, devido a problemas de saúde. Além da partilha da experiência do uso, a fala dos participantes seguiu no sentido para entender melhor como o medicamento atuava em seu corpo e quais processos desencadeava, de acordo com a experiência vivida. A conversa seguiu também no sentido de discutir os efeitos negativos para a saúde do uso prolongado de opioides sem acompanhamento médico. Foi ainda um momento para discutir dúvidas e curiosidades, o que tornou a atividade uma experiência gratificante e motivadora.

Esse resultado obtido vem de encontro ao proposto por Freire (2011), demonstrando que a roda de conversa proporciona situações de ensino que oportunizam aos estudantes direcionar sua própria aprendizagem. Cabe ressaltar que a aprendizagem possibilitada com a atividade foi tanto nos aspectos pedagógicos quanto nos aspectos científicos. A estratégia mostrou-se facilitadora da aprendizagem, além de motivar os estudantes a buscar mais informações sobre o assunto.

Revista Extensão em Foco, nº 19, Jul./ Dez. (2019), p. 104 - 114. 
A análise dos resultados relativos às repostas dadas aos questionários, antes do início e após o minicurso (FIGURA 1), demonstrou diferença significativa $(p<0,05)$ entre a porcentagem de respostas corretas para as questões 1,2 e 3, tendo sido obtidas porcentagens de acertos menores antes do minicurso do que após a realização deste. Este resultado pode indicar que muitos participantes não fizeram uso do material didático disponibilizado antes do minicurso, pois se o tivessem feito, certamente, a diferença entre as porcentagens de respostas corretas antes e após o minicurso não seriam significativas ( $\mathrm{p}>0,05)$. Por outro lado, este dado sugere também que a estratégia utilizada no minicurso possibilitou a compreensão do tema estudado, tendo em vista o aumento de respostas corretas obtidas no questionário respondido após o minicurso. Como pode ser observado na Figura 2, houve aumento significativo $(\mathrm{p}<0,05)$ do total de respostas corretas após o minicurso, enfatizando que a estratégia utilizada pode ser uma ótima opção para o ensino superior.

Figura 1 - Respostas certas (\%) obtidas para cada questão dos questionários aplicados antes e após o minicurso

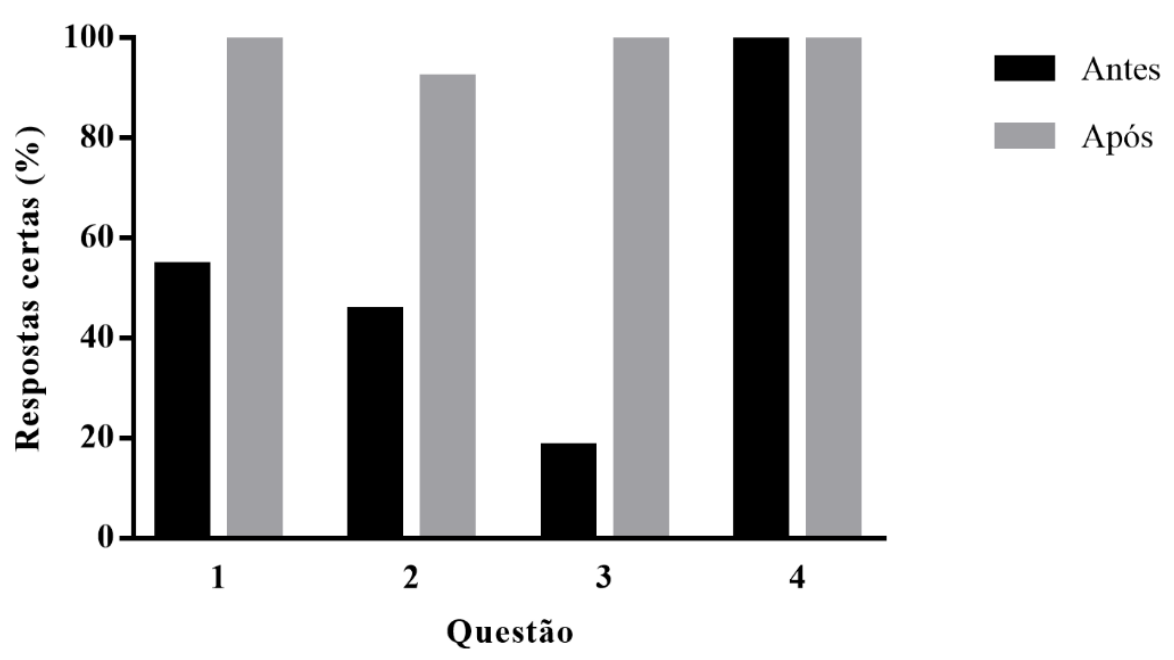

Fonte: Dados dos autores.

Revista Extensão em Foco, nº 19, Jul./ Dez. (2019), p. 104 - 114. 
Figura 2 - Total de respostas certas (\%) obtidas para as questões dos questionários aplicados antes e após o minicurso

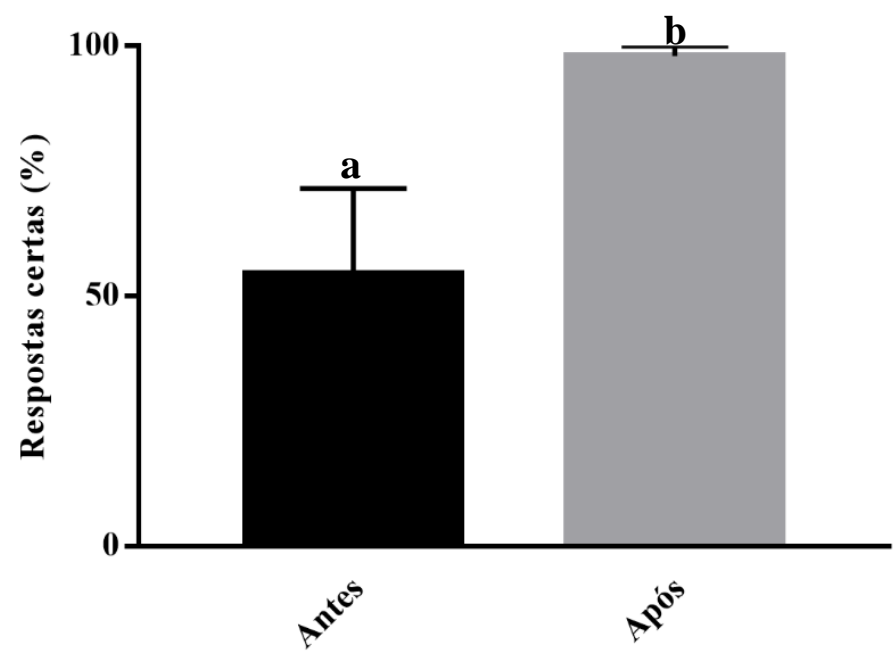

Fonte: Dados dos autores.

Letras diferentes indicam diferença significativa $(\mathrm{p}=0,04)$ pelo teste $t$ - Student

Sendo assim, o minicurso parece uma boa estratégia para ensino e aprendizagem de temas diversos, assim, como a roda de conversa, que por meio do diálogo permite a reflexão capaz de levar à compreensão e reelaboração de conceitos e conhecimentos e pode contribuir para a articulação entre experiências pessoais e profissionais, contribuindo para a sólida formação universitária e comprometida com a transformação da realidade.

\section{CONCLUSÕES}

O uso e abuso de drogas é um problema mundial e apesar de abordado em cursos da área de saúde é ainda pouco estudado nos demais cursos. O presente trabalho evidenciou a utilização de minicurso e de roda de conversa como estratégias didáticas eficientes para a primeira abordagem e ampliação dos conhecimentos sobre opioides fora da sala de aula. Estas estratégias de ensino utilizadas no ensino superior proporcionaram grande troca de 
aprendizado e conhecimento entre os participantes. Assim, parecem ser boa opção para disseminar o conhecimento de temas não contemplados pelos currículos ou mesmo para temas curriculares, pois contribuem para a autonomia do aprendizado dos estudantes e oportunizam uma forma para que o aluno participe ativamente da construção do conhecimento.

\section{AGRADECIMENTOS}

Ao PET-MEC-SESu pela concessão de bolsa aos autores.

\section{REFERÊNCIAS BIBLIOGRÁFICAS}

BORGES, Antonio Tarciso. Novos rumos para o laboratório escolar de ciências. Caderno Brasileiro de Ensino de Física, v. 19, n. 3, p. 291-313, 2002.

CARVAlHO, Anna Maria Pessoa de; GIL-PÉREZ, Daniel. 10. ed. Formação de professores de ciências: tendências e inovações. São Paulo: Cortez, 2011.

FREIRE, Paulo. Pedagogia da autonomia: saberes necessários à prática educativa. 25. ed. São Paulo: Paz e Terra, 2011.

JORGE, Kelly Oliva; FERREIRA, Raquel Conceição; FERREIRA, Efigênia Ferreira e; KAWACHI, Ichiro; ZARZAR, Patrícia Maria; PORDEUS, Isabela Almeida. Influência do grupo de pares e uso de drogas ilícitas entre adolescentes brasileiros: um estudo transversal. Cadernos de Saúde Pública, v. 34, n. 3, e00144316, 2018.

KRAYCHETE, Durval Campos; SIQUEIRA, José Tadeu Tesseroli de; ZAKKA, Telma Regina Mariotto; GARCIA, João Batista Santos. Recomendações para uso de opioides no Brasil: Parte III. Uso em situações especiais (dor pósoperatória, dor musculoesquelética, dor neuropática, gestação e lactação). Revista Dor, v. 15, n. 2, p. 126-132, 2014.

KRASILCHIK, Myriam. Prática de Ensino de Biologia. 4. ed. São Paulo: Editora da Universidade de São Paulo, 2008.

NAHAS, Miriam Almeida; MELO, Ana Paula Souto; COURNOS, Francine; MCKINNON, Karen; WAINBERG, Milton; GUIMARÃES, Mark Drew Crosland. Recent illicit drug use among psychiatric patients in Brazil: a national representative study. Revista de Saúde Pública, v. 51, p. 74, 2017.

NASCIMENTO, Daiana Ciléa Honorato; SAKATA, Rioko Kimiko. Dependência de opioide em pacientes com dor crônica. Revista Dor, v. 12, n. 2, p. 160-165, 2011.

Revista Extensão em Foco, nº 19, Jul./ Dez. (2019), p. 104 - 114. 
PEREIRA, Mariana de Moura; ANDRADE, Letycia de Paiva; TAKITANE, Juliana. Evolução do uso abusivo de derivados de ópio. Saúde, Ética e Justiça, v. 21, n. 1, p. 12-17, São Paulo, 2016.

SHOR, Ira; FREIRE, Paulo. What is the "dialogical method" of teaching? Journal of Education, v. 169, n. 3, p. 11-31, 1987.

UNODC (United Nations Office on Drugs and Crime). World Drug Report 2017. Viena: Nações Unidas, 2017.

WARSCHAUER, Cecília. Rodas em rede: oportunidades formativas na escola e fora dela. Rio de Janeiro: Paz e Terra, 2001.

WARSCHAUER, Cecília. A roda e o registro: uma parceria entre professor, aluno e conhecimento. Rio de Janeiro: Paz e Terra, 2002.

WARSCHAUER, Cecília. Rodas e narrativas: caminhos para a autoria de pensamento, para a inclusão e a formação. Roda e Registro - desenvolvimento pessoal e profissional. In SCOZ, B. (org) Psicopedagogia: contribuições para a educação pós-moderna. Petrópolis: Vozes, 2004. p. 13-23.

WEBSTER, Lynn. Risk factors for opioid-use disorder and overdose. Anesthesia e Analgesia, v. 125, n. 5, p. 1741-1748, 2017.

Revista Extensão em Foco, nº 19, Jul./ Dez. (2019), p. 104 - 114. 
Revista Extensão em Foco, no 19, Jul./ Dez. (2019), p. 104 - 114. 